\title{
Ambient Temperature and Cardiac Accumulation of ${ }^{18}$ F-FDG
}

\author{
Samantha O’Loughlin ${ }^{1}$, Geoffrey M. Currie ${ }^{1,2}$, Marko Trifonovic ${ }^{3}$, and Hosen Kiat ${ }^{1,2,4}$ \\ ${ }^{I}$ Faculty of Science, Charles Sturt University, Wagga Wagga, New South Wales, Australia; ${ }^{2}$ Australian School of Advanced Medicine, \\ Macquarie University, Sydney, New South Wales, Australia; ${ }^{3}$ Macquarie Medical Imaging, Macquarie University Hospital, Sydney, \\ New South Wales, Australia; and ${ }^{4}$ Cardiac Health Institute, Sydney, New South Wales, Australia
}

\begin{abstract}
Warming patients by changing the ambient environment (rather than core temperature) has been reported to reduce brown adipose tissue activity, thereby reducing artifacts in ${ }^{18} \mathrm{~F}-\mathrm{FDG}$ PET. Nonetheless, a reduction in cardiac uptake of ${ }^{18} \mathrm{~F}-\mathrm{FDG}$ has been incidentally noted during ambient warming. This study examined the impact of seasonal variations in ambient temperatures on cardiac uptake of ${ }^{18} \mathrm{~F}-\mathrm{FDG}$. Methods: Consecutive ${ }^{18}$ F-FDG PET patients were recruited into summer and winter cohorts. The protocol was highly standardized; however, data were excluded when scanning took place other than 60 min after injection. Mean and maximum counts per pixel in the cardiac region and cardiac standardized uptake value were determined and correlated with the daily minimum, maximum, and mean temperature (day of scan), mean temperature on the scan day and preceding $2 \mathrm{~d}$ (3-d window), and mean temperature 1, 2, 3, and 4 wk before scanning. Results: No statistically significant differences were noted between cohorts (summer and winter) for age, sex, weight, height, or dose. As expected, the summer cohort was associated with statistically higher minimum, maximum, and mean temperatures, longer days, and greater sunlight hours (all $P<0.001$ ). The mean and maximum heart counts were statistically higher in winter than summer $(P=$ 0.031 and $P=0.024$, respectively). The cardiac standardized uptake value was statistically higher for winter than summer $(P=0.026)$. The key factors in cardiac accumulation of ${ }^{18} \mathrm{~F}-$ FDG were the minimum temperature on the day of the scan and the 3-d mean temperature. Conclusion: Cardiac accumulation of ${ }^{18} \mathrm{~F}-\mathrm{FDG}$ is influenced by the ambient temperature, in particular the minimum temperature on the day of the scan and the 3-d average temperature before the scan. Further investigation is warranted to examine the impact of this observation on clinical protocols and cardiac ${ }^{18} \mathrm{~F}-\mathrm{FDG}$ PET study results.
\end{abstract}

Key Words: ambient temperature; cardiac; ${ }^{18}$ F-FDG PET

J Nucl Med Technol 2014; 42:188-193

DOI: 10.2967/jnmt.114.143867

$\mathbf{P}_{\mathrm{H}}$ ET with the glucose analog tracer ${ }^{18} \mathrm{~F}-\mathrm{FDG}$ evaluates cellular metabolism and is commonly used for the detection and staging of disease in oncology patients $(1-3) .{ }^{18} \mathrm{~F}-\mathrm{FDG}$

\footnotetext{
Received May 28, 2014; revision accepted Jul. 7, 2014.

For correspondence or reprints contact: Geoffrey M. Currie, Charles Sturt

University, P.O. Box U102, CSU, Wagga Wagga, NW 2650, Australia.

E-mail: gcurrie@csu.edu.au

Published online Aug. 7, 2014

COPYRIGHT (C 2014 by the Society of Nuclear Medicine and Molecular Imaging, Inc.
}

PET is also widely used to assess myocardial viability $(4,5)$. ${ }^{18}$ F-FDG PET in oncology has been reported to confront issues with artifactual accumulation of ${ }^{18} \mathrm{~F}-\mathrm{FDG}$ in brown adipose tissue (BAT) (6). Warming patients by changing the ambient environment (rather than core temperature) has been reported to successfully reduce BAT activity; however, one recent report noted that warming patients also reduced cardiac uptake of ${ }^{18}$ F-FDG (Fig. 1) (6). Although this observation may relate to seasonal variations in cardiac event rates, there may also be important implications for cardiac ${ }^{18}$ F-FDG PET diagnostic integrity. Cardiac glucose metabolism depends on numerous factors including substrate availability, cardiac workload, myocardial perfusion, and medication (7). Increased sympathetic activity drives increased metabolic demand for glucose $(8,9)$, and although the connection has yet to be reported in the literature, this investigation hypothesized that the increased sympathetic activity associated with cooler ambient temperatures results in comparatively increased cardiac ${ }^{18} \mathrm{~F}-\mathrm{FDG}$ uptake over a warmer ambient temperature.

\section{MATERIALS AND METHODS}

\section{Patient Data}

Consecutive patients undergoing whole-body oncologic ${ }^{18} \mathrm{~F}$ FDG PET scans were recruited for a summer period $(n=25)$ and subsequent winter period $(n=37)$. PET scanning was performed using a Discovery 64-slice time-of-flight PET/CT scanner (GE Healthcare) in environmental conditions (room temperature) identical between the 2 cohorts (internal climate control). A highly standardized protocol was used in which patients fasted for at least $4 \mathrm{~h}$ before the PET procedure. A fasting blood sample was taken from patients, and the blood glucose level was adjusted so that it was within the reference range. Patients were well hydrated before the procedure. Intravenous injection of ${ }^{18} \mathrm{~F}-\mathrm{FDG}$ ranged between 208 and $370 \mathrm{MBq}$ according to patient weight, with a mean dose of $271 \mathrm{MBq}$. A 60-min isolated and relaxed radiopharmaceutical uptake phase followed. Data were excluded when scanning took place other than 60 min after injection because of the impact such variation has on standardized uptake value (SUV) calculation. Whole-body imaging was performed from the vertex to below the knees. A diagnostic-quality, contrast-enhanced CT scan was obtained through the brain, chest, abdomen, and pelvis, with lowdose CT imaging performed elsewhere for attenuation correction and lesion localization. PET images were reconstructed using non-breath-hold CT-based attenuation correction with orderedsubset expectation maximization iterative reconstruction for a 4-mm slice thickness. Mean and maximum counts per pixel 


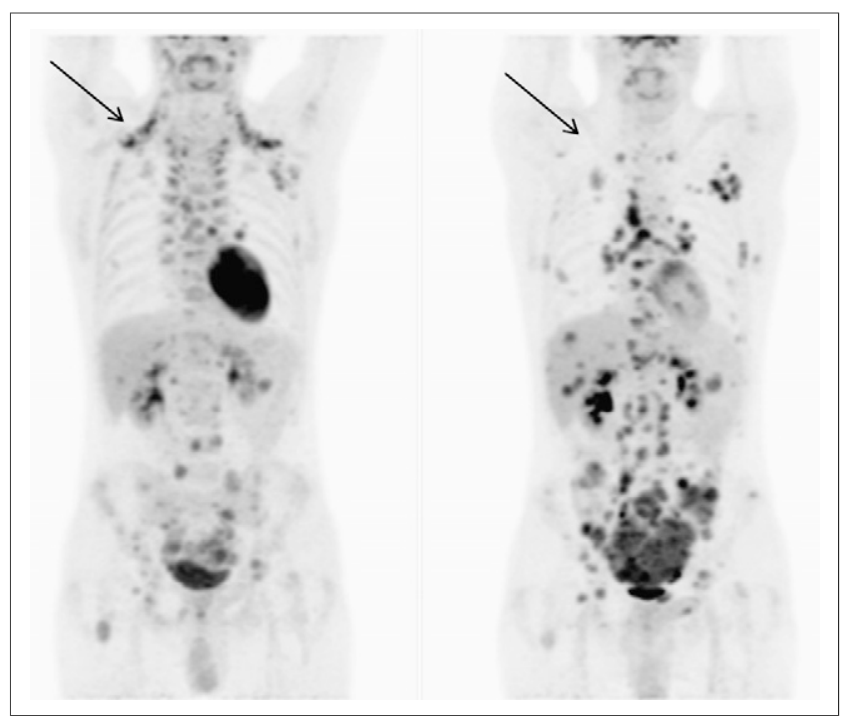

FIGURE 1. PET scans in same patient under different ambient temperatures showing reduction of BAT in warm temperatures (right) and intense cardiac ${ }^{18} \mathrm{~F}-\mathrm{FDG}$ uptake in cooler conditions (left) (6).

and SUV in the cardiac and liver regions were determined using whole-heart regions of interest around mid-coronal slices. Cardiac ${ }^{18}$ F-FDG SUV was determined as injected dose (MBq) divided by weight $(\mathrm{kg})$, subsequently divided into the average heart counts per pixel. Patient age, height, weight, sex, and dose were recorded. The project was approved by an appropriate institutional committee of ethics in human research.

\section{Meteorologic Data}

According to the patient scan date, meteorology records (public data) were accessed to obtain weather details for the region serviced by the imaging department. The mean, maximum, and minimum daily temperatures were recorded for the day of each patient procedure and for the preceding $2 \mathrm{~d}, 1 \mathrm{wk}, 2 \mathrm{wk}, 3$ wk, and $4 \mathrm{wk}$. The daily global solar exposure leading up to each scan date was available in $\mathrm{kWh} / \mathrm{m}^{2}$ for the same period. Length of daily sun time was calculated using data on time of sunrise and sunset.

TABLE 1

Summary of Key Variables in Summer and Winter

\begin{tabular}{lccc}
\hline \multicolumn{1}{c}{ Variable } & Summer & Winter & $P$ \\
\hline Dose (mean) & $274.6 \mathrm{MBq}$ & $269.0 \mathrm{MBq}$ & 0.533 \\
Age (mean) & $68.3 \mathrm{y}$ & $67.2 \mathrm{y}$ & 0.738 \\
Sex & $38.9 \%$ female & $44.4 \%$ female & 0.712 \\
$\begin{array}{c}\text { Average liver } \\
\text { counts per pixel } \\
\text { (mean) }\end{array}$ & 2.92 & 3.17 & 0.495 \\
$\begin{array}{c}\text { Maximum liver } \\
\text { counts per } \\
\text { pixel (mean) }\end{array}$ & 5.37 & & \\
$\begin{array}{c}\text { Average heart } \\
\text { counts per } \\
\text { pixel (mean) }\end{array}$ & 3.00 & 5.35 & 0.979 \\
$\begin{array}{c}\text { Maximum heart } \\
\text { counts per } \\
\text { pixel (mean) }\end{array}$ & 5.36 & 4.11 & 0.031 \\
\hline
\end{tabular}

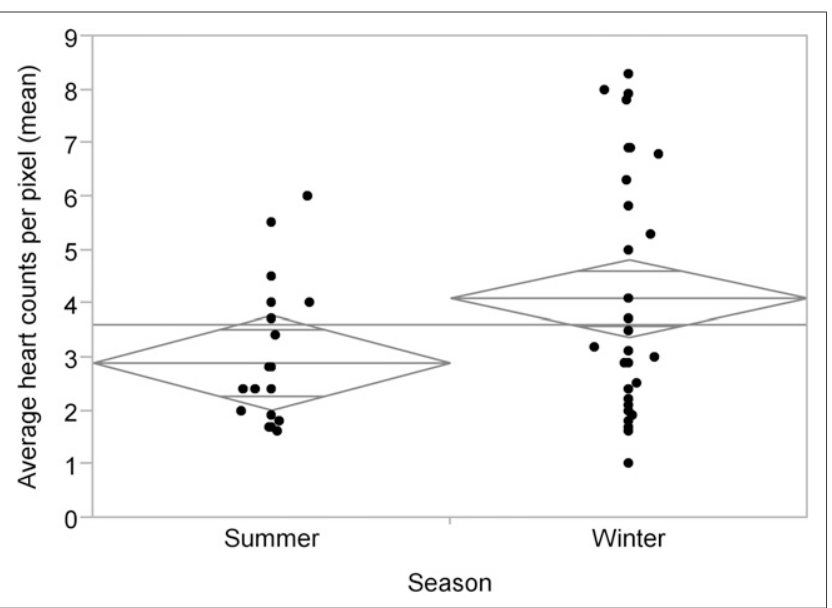

FIGURE 2. Average heart counts per pixel vs. season $(P=$ 0.031). Diamonds represent $95 \% \mathrm{Cl}$ of each mean (center line of diamond), and horizontal line represents overall mean.

\section{Statistical Analysis}

Counts per pixel and SUV data were correlated with the season plus the daily minimum, maximum, and mean temperature (day of scan), mean temperature on the scan day and preceding $2 \mathrm{~d}$ (3d mean temperature), and mean temperature $1,2,3$, and 4 wk before scanning. Student $t$ testing was used to assess continuous data. The Pearson $\chi^{2}$ test was used for categoric data with normal distribution, and the likelihood ratio $\chi^{2}\left(\mathrm{G}^{2}\right)$ test was used for categoric data without normal distribution. A $P$ value of less than 0.05 was considered significant. Normality of distribution was determined using the Shapiro-Wilk W test, with a $P$ value of less than 0.05 indicating that the data vary significantly from normality.

\section{RESULTS}

After exclusion of patients whose scanning took place other than 60 min after injection, there were 45 patients: 18 in summer and 27 in winter. There was no statistically significant variation in this distribution from a hypothetical even split $(P=0.180)$. Men represented 57.8\% $(n=26)$ of the study sample $(P=0.297)$. The mean age was $67.7 \mathrm{y}$

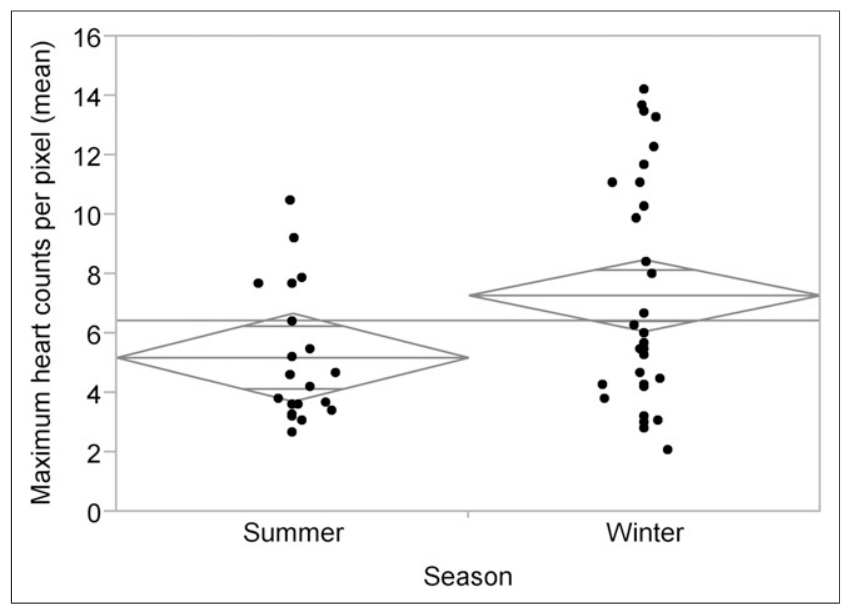

FIGURE 3. Maximum heart counts per pixel vs. season $(P=$ 0.024). Diamonds represent $95 \% \mathrm{Cl}$ of each mean (center line of diamond), and horizontal line represents overall mean. 
TABLE 2

Meteorologic Data for Summer and Winter

\begin{tabular}{lcrc}
\hline \multicolumn{1}{c}{ Temperature } & Summer & Winter & $P$ \\
\hline Mean daily maximum & $27.9^{\circ} \mathrm{C}$ & $17.5^{\circ} \mathrm{C}$ & $<0.001$ \\
Mean daily minimum & $21.0^{\circ} \mathrm{C}$ & $9.8^{\circ} \mathrm{C}$ & $<0.001$ \\
Daily average & $24.4^{\circ} \mathrm{C}$ & $13.7^{\circ} \mathrm{C}$ & $<0.001$ \\
3-d mean & $24.6^{\circ} \mathrm{C}$ & $14.1^{\circ} \mathrm{C}$ & $<0.001$ \\
1-wk mean & $24.9^{\circ} \mathrm{C}$ & $14.0^{\circ} \mathrm{C}$ & $<0.001$ \\
2-wk mean & $24.6^{\circ} \mathrm{C}$ & $13.8^{\circ} \mathrm{C}$ & $<0.001$ \\
3-wk mean & $24.4^{\circ} \mathrm{C}$ & $13.8^{\circ} \mathrm{C}$ & $<0.001$ \\
4-wk mean & $24.2^{\circ} \mathrm{C}$ & $13.9^{\circ} \mathrm{C}$ & $<0.001$ \\
\hline
\end{tabular}

(95\% confidence interval [CI], 64.4-70.9 y), with a normally distributed $(P=0.434)$ range of $47-89 \mathrm{y}$. The mean ${ }^{18} \mathrm{~F}-\mathrm{FDG}$ dose was $271.2 \mathrm{MBq}$ (95\% CI, 262.4-280.0 $\mathrm{MBq}$ ), with a range of 208-370 MBq.

With respect to the distribution of variables between the summer and winter cohorts, there were no statistically significant differences noted for sex $(P=0.712)$, age $(P=$ $0.738)$, or patient dose $(P=0.533)$ (Table 1). No statistically significant differences were noted in the average liver counts per pixel $(P=0.495)$ or the maximum liver counts per pixel $(P=0.979)$ between summer and winter. Importantly, there was a statistically significant increase from winter to summer in the average heart counts per pixel $(P=$ $0.031)$ (Fig. 2) and the maximum heart counts per pixel $(P=$ 0.024) (Fig. 3).

Not surprisingly, there were statistically significant increases in summer compared with winter for meteorologic data (all $P<0.001$ ) (Table 2). Weak statistically significant correlations were noted between the day length (sunlight hours) and both the average counts per pixel in the heart $(P=$ $0.0354)$ and the maximum counts per pixel in the heart $(P=$ 0.0248 ). Increasing counts per pixel corresponded with shorter days. No statistically significant correlation was found between either the average counts per pixel in the heart or maximum counts per pixel in the heart against both daily maximum temperature and the average daily temperature $(P>0.05)$. There were, however, weak statistically significant correlations noted between the daily minimum temperature and both the average counts per pixel in the heart $(P=0.0476)$ and the maximum counts per pixel in the heart $(P=0.0437)$. Higher counts per pixel corresponded to lower minimum daily tem- peratures (Fig. 4). A weak statistically significant correlation was also noted between the 3-d mean temperature and both the average counts per pixel in the heart $(P=0.0475)$ and maximum counts per pixel in the heart $(P=0.0408)$. Higher counts per pixel corresponded to lower temperatures (Fig. 5). There were, however, no statistically significant correlations observed between average or maximum counts per pixel in the heart and mean temperatures for $1,2,3$, or 4 wk before the study (all $P>0.05$ ) (Table 3 ).

Height, weight, and dose were normally distributed. No statistically significant difference was noted between the mean height of the summer cohort $(172.1 \mathrm{~cm} ; 95 \% \mathrm{CI}$, 167.3-176.9) and the winter cohort $(168.5 \mathrm{~cm} ; 95 \% \mathrm{CI}$, 164.6-172.5) $(P=0.2557)$. No statistically significant difference was noted between the mean weight of the summer cohort $(79.4 \mathrm{~kg}$; 95\% CI, 71.0-87.8) and the winter cohort (77.7 kg; 95\% CI, 70.9-84.6) $(P=0.7657)$. No statistically significant difference was noted between the mean patient dose for the summer cohort (274.6 MBq; 95\% CI, 260.6288.7) and the winter cohort (269.0; 95\% CI, 257.5-280.4) $(P=0.5334)$. There was, however, a statistically significant difference between the cardiac SUV calculated for the summer cohort $(0.83 ; 95 \% \mathrm{CI}, 0.49-1.17)$ and the winter cohort (1.27; 95\% CI, 0.99-1.55) $(P=0.0256)$ (Fig. 6).

As expected, a statistically significant correlation was observed between patient weight and dose $(P<0.001)$, with an increase in dose corresponding to an increase in weight $\left(R^{2}=0.403\right)$. A statistically significant but weak correlation was noted between patient weight and height $(P<0.001)$, with an increasing weight corresponding to an increasing height $\left(R^{2}=0.287\right)$. As expected, a weak but statistically significant correlation was noted between patient height and dose $(P=0.0145)$, with an increase in dose corresponding to an increase in height $\left(R^{2}=0.118316\right)$. A statistically significant but weak correlation was noted between total day length and cardiac SUV $(P=0.0224)$, with a decrease in day length corresponding to an increase in cardiac SUV $\left(R^{2}=0.1039\right)$. No statistically significant correlation was found between cardiac SUV and the daily maximum temperature $(P=0.1116)$. A statistically significant but weak inverse correlation was noted between temperature and cardiac SUV, with an increase in cardiac SUV corresponding to a decrease in daily minimum temperature $(P=0.0244$,
FIGURE 4. Average heart counts per pixel (left) and maximum heart counts per pixel (right) plotted against minimum daily temperature showing decreasing counts per pixel with increasing daily minimum temperatures $(P=0.0476$, $R^{2}=0.09$, and $P=0.0475, R^{2}=0.09$, respectively).

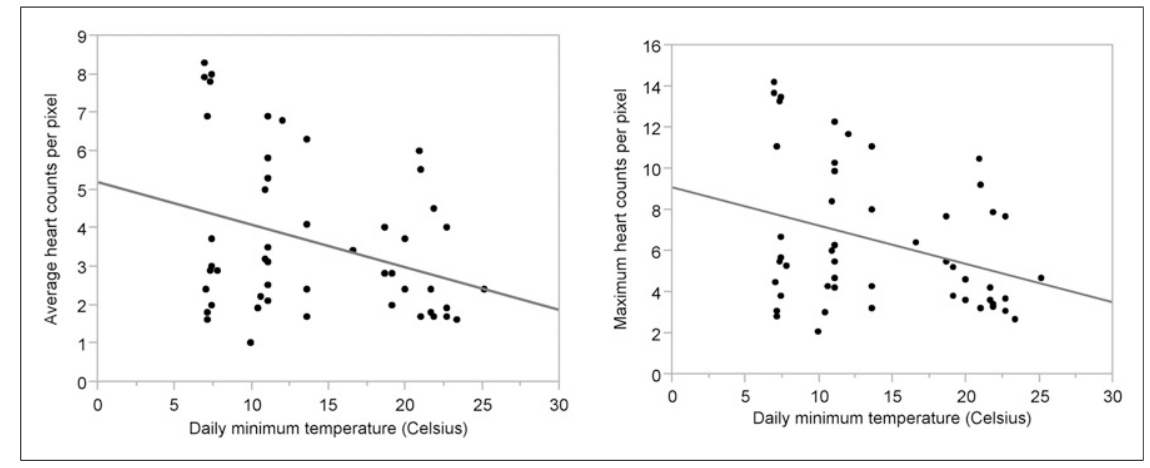



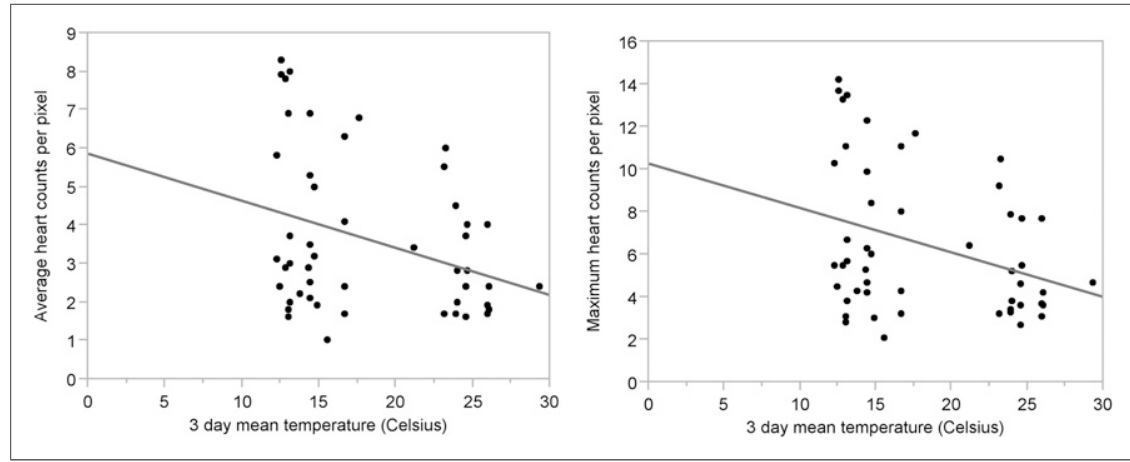

FIGURE 5. Average heart counts per pixel (left) and maximum heart counts per pixel (right) plotted against 3-d mean temperature showing decreasing counts per pixel with increasing 3-d mean temperatures $\left(P=0.0437, R^{2}=0.09\right.$, and $P=0.0408, R^{2}=0.09$, respectively).

$\left.R^{2}=0.1011\right)$ (Fig. 7), daily average temperature $(P=$ $\left.0.0455, R^{2}=0.0809\right)$, and 3 -d mean temperature $(P=$ $0.0320, R^{2}=0.1039$ ) (Fig. 7). In contrast, there were no statistically significant correlations between cardiac SUV and mean temperatures for 1, 2, 3, or $4 \mathrm{wk}$ before the study (all $P>0.05$ ) (Table 3).

\section{DISCUSSION}

To our knowledge, this study was the first to formally examine the impact of seasonal and environmental temperature on cardiac ${ }^{18}$ F-FDG uptake. The results are concordant with the incidental cardiac ${ }^{18} \mathrm{~F}$-FDG finding reported in the previous study of thermal control of BAT in oncologic ${ }^{18}$ F-FDG PET (6). Average heart counts per pixel, maximum heart counts per pixel, and cardiac SUV were all found to be statistically higher when measured during winter. Furthermore, average heart counts per pixel, maximum heart counts per pixel, and cardiac SUV showed statistically significant relationships with daily minimum temperature and 3-d mean temperature. The lack of statistically significant correlation with maximum temperature is likely to reflect the maximum's being more of a daily spike than a sustained ambient environment. Moreover, patients tend to present to departments in the morning, before the peak heat of the day has been reached. These results may also suggest that the key issue is not warming the patient to decrease the uptake but rather cooling the patient to increase the uptake; much like BAT increasing in appearance on ${ }^{18}$ F-FDG imaging.

Lower seasonal ambient temperatures provoke a range of physiologic responses including peripheral vasoconstriction, and increased cardiac sympathetic activity, heart rate, diastolic blood pressure, myocardial oxygen demand, blood glucose concentration, and plasma noradrenaline concentration $(8,10-12)$. These results suggest that exposure to cool or cold conditions would increase ${ }^{18} \mathrm{~F}-\mathrm{FDG}$ accumulation in the myocardium whereas, in contrast, warmer ambient temperatures would decrease cardiac sympathetic activity and ${ }^{18} \mathrm{~F}$-FDG uptake. The absence of a statistically significant relationship between uptake and longer durations before the scan (1-4 wk) is likely to reflect a dilution effect of variations in temperature across the weeks before the scans combined with the power of the acute effects of the specific daily minimum temperature and potential discordance with the longer average temperatures. For example, despite strong correlations between the minimum temperature on the day of the scan and the daily averages for 1 through $4 \mathrm{wk}\left(P<0.001\right.$ and $R^{2}$ in excess of 0.8$)$, this correlation reflects the general upward trend from winter to summer. When the relationship is isolated by season, there is no correlation between the daily minimum temperature and the average temperatures for the 1 - to 4 -wk period $(P>$ 0.8 and $\left.R^{2}<0.1\right)$.

Warming interventions for oncologic studies appear advantageous to eliminate BAT accumulation. Reduction of cardiac ${ }^{18} \mathrm{~F}$-FDG uptake during warming might also allow for improved physiologic assessment of the intrathoracic organs. Nonetheless, warming patients would be unhelpful in cardiac ${ }^{18} \mathrm{~F}$-FDG studies and, probably more applicable to this cohort, the ambient temperatures require standardization at a cool temperature.

These results indicate that the ambient temperature and seasonal variations influence cardiac ${ }^{18} \mathrm{~F}$-FDG uptake, meaning that it may be advantageous to acclimatize patients in

TABLE 3

Summary of Relationship Between Cardiac ${ }^{18}$ F-FDG Uptake SUV and Various Ambient Temperatures

\begin{tabular}{lccl}
\hline \multicolumn{1}{c}{ Temperature } & Average heart counts per pixel & Maximum heart counts per pixel & Cardiac SUV \\
\hline Daily minimum & $P=0.0476, R^{2}=0.09$ & $P=0.0437, R^{2}=0.09$ & $P=0.0244, R^{2}=0.10$ \\
Daily maximum & $P=0.1674$ & $P=0.1446$ & $P=0.1116$ \\
Daily mean & $P=0.0820$ & $P=0.0721$ & $P=0.0455, R^{2}=0.08$ \\
3-d mean & $P=0.0475, R^{2}=0.09$ & $P=0.0408, R^{2}=0.09$ & $P=0.0320, R^{2}=0.10$ \\
1-wk mean & $P=0.0765$ & $P=0.0687$ & $P=0.0593$ \\
2-wk mean & $P>0.08$ & $P=0.0781$ & $P>0.08$ \\
3-wk mean & $P>0.08$ & $P=0.0817$ & $P>0.08$ \\
4-wk mean & $P>0.08$ & $P=0.0830$ & $P>0.08$ \\
\hline
\end{tabular}




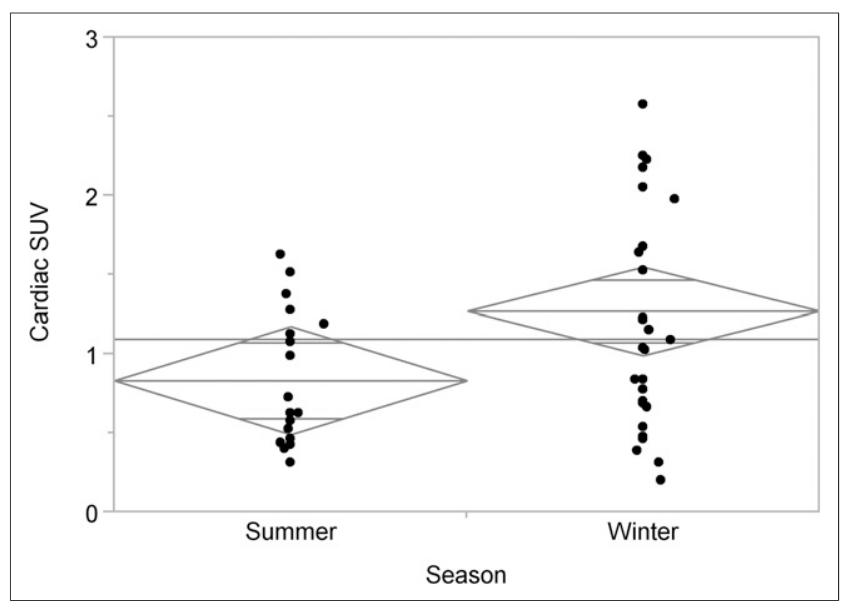

FIGURE 6. Cardiac SUV vs. season $(P=0.026)$. Diamonds represent $95 \% \mathrm{Cl}$ of each mean (center line of diamond), and horizontal line represents overall mean.

a cooler environment before and during the uptake phase. It is also worth considering how patients dress during colder months. Overcompensation for the cold with heating and layers of clothes may produce a warming effect during winter. Given the variation in cardiac uptake of ${ }^{18} \mathrm{~F}-\mathrm{FDG}$, a standardized protocol should include controlled ambient temperatures not only during uptake but also for at least $1 \mathrm{~h}$ before intravenous administration of ${ }^{18} \mathrm{~F}-\mathrm{FDG}$. This study was undertaken in a relatively temperate climate in which winter temperatures are not typically too cold. A similar investigation in very cold winter environments might yield paradoxic results. Indeed, further investigation in cohorts from climates on either end of the spectrum is recommended. Further investigation is also required to determine whether the thermal gradient between warm and cold is the same for both normal and diseased myocardium. Clearly, this may have significant implications for diagnostic integrity if a differential response between normal and diseased myocardium is revealed. It is also important to consider and further evaluate the impact of glucose-loading protocols. The effect of insulin driving cardiac ${ }^{18} \mathrm{~F}$-FDG uptake may overcome or succumb to the influences of ambient temperature.

Seasonal variation has been reported in various diseases and conditions. Winter is associated with an increased risk of cardiovascular events, morbidity, and mortality due to the environmental temperature $(8,9)$. Exposure to lower temperatures has been linked to induction of cardiac electrical instability, ventricular arrhythmias, myocardial infarction, acute ischemia, peripheral vasoconstriction, sudden cardiac death, and deep venous thrombosis $(8,9)$. The increased sympathetic activity in cooler ambient temperatures is likely to drive both cardiovascular risk and increased metabolic demand for glucose.

In cardiac tissue, sympathetic innervation of autonomic nerves increases cardiac contractility, leading to an increased heart rate. This is mediated by the highly abundant postsynaptic myocardial $\beta$-adrenergic receptors in response to cold temperatures. The sympathetic nervous system is significant in cardiovascular homeostasis through innervation of heart, kidney, blood vessels, and differing neurotransmitters (norepinephrine and acetylcholine) (13-15). Cold temperatures initiate excitability of cardiomyocytes through increased sinoatrial node discharge, reducing the conduction time of cardiac fibers and increasing cell permeability to calcium, which in turn increases cardiac contractility force and heart rate (13). It is important to draw a distinction between what is being understood about the impact of ambient temperature on physiologic processes and what has been widely reported regarding the impact of changes to the core body temperature. The process, physiology, outcomes, and implications vary markedly.

There are several patient-related factors that can affect cardiac ${ }^{18}$ F-FDG uptake. The cardiac SUV has been reported to increase in patients with heart failure, patients taking benzodiazepines, men younger than $30 \mathrm{y}$ old, and patients who have fasted for less than $5 \mathrm{~h}$ (7). Conversely, significantly lower cardiac ${ }^{18} \mathrm{~F}-\mathrm{FDG}$ uptake has been observed in patients taking bezafibrate or levothyroxine and those who are diabetic (7). Although not all of these variables were recorded for this study, it is believed that random distribution between the 2 cohorts eliminates this factor as a major limitation. It is, however, important from the context of protocol standardization that medications altering the biodistribution of a radiopharmaceutical, particularly the target organ, be managed. Interestingly, an alternative approach to thermal control of BAT has been the use of pharmaceuticals, including propranolol, reserpine, diazepam, and fentanyl. Although each has been reported to be effective (6), their mechanism of
FIGURE 7. Cardiac SUV plotted against minimum daily temperature (left) and 3-d mean temperature (right) showing decreasing cardiac SUV with increasing temperatures $\left(P=0.0244, R^{2}=0.10\right.$, and $P=0.0320, R^{2}=0.10$, respectively).
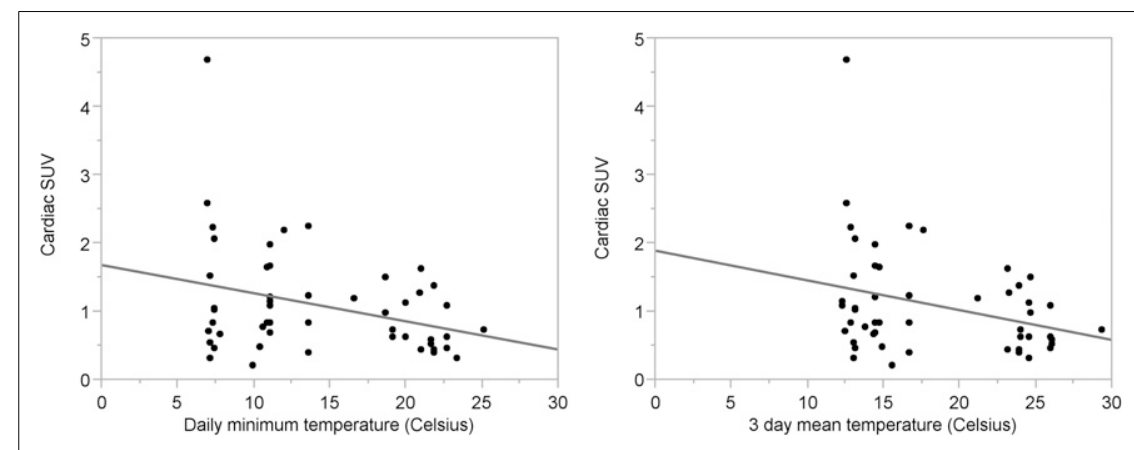
action and degree of success vary. In a key study examining the effects of propranolol, reserpine, and diazepam on BAT activity, all 3 were shown to reduce BAT by a statistically significant degree; however, diazepam was the least effective by a large margin (16). In the same study, propranolol and reserpine produced a statistically significant reduction in cardiac uptake of ${ }^{18} \mathrm{~F}-\mathrm{FDG}$ ( $>50 \%$ reduction) whereas diazepam produced a small increase in ${ }^{18} \mathrm{~F}-\mathrm{FDG}$ uptake (16). This observation is concordant with the findings for diazepam by Israel et al. (7). Further investigation is recommended to more thoroughly inform standardization of cardiac ${ }^{18} \mathrm{~F}$-FDG patient preparation.

\section{CONCLUSION}

These results indicate that the average heart counts per pixel, maximum heart counts per pixel, and cardiac SUV are significantly increased in response to decreasing daily minimum temperature and decreasing 3-d mean temperature. Cardiac uptake of ${ }^{18} \mathrm{~F}-\mathrm{FDG}$ is significantly increased during the winter months, compared with summer. Patient ambient temperature before ${ }^{18} \mathrm{~F}$-FDG administration plays a key role in radiopharmaceutical localization, and consideration needs to be given to standardization of patient preparation in this regard. Further investigation is recommended to evaluate this relationship with respect to more extreme climates and differential response between normal and diseased tissue, and for insight into seasonal variations in cardiac disease.

\section{DISCLOSURE}

No potential conflict of interest relevant to this article was reported.

\section{REFERENCES}

1. Fletcher JW, Djulbegovic B, Soares HP, et al. Recommendations on the use of ${ }^{18}$ F-FDG PET in oncology. J Nucl Med. 2008;49:480-508.

2. Pauwels EKJ, Coumou AW, Kostkiewicz M, Kairemo K. $\left[{ }^{18}\right.$ F $]$ fluoro-2-deoxy-Dglucose positron emission tomography/computed tomography imaging in oncology: initial staging and evaluation of cancer therapy. Med Princ Pract. 2013; 22:427-437.

3. Tsurusaki M, Okada M, Kuroda H, Matsuki M, Ishii K, Murakami T. Clinical application of ${ }^{18} \mathrm{~F}$-fluorodeoxyglucose positron emission tomography for assessment and evaluation after therapy for malignant hepatic tumor. J Gastroenterol. 2014;49:46-56.

4. Allman KC. Noninvasive assessment myocardial viability: current status and future directions. J Nucl Cardiol. 2013;20:618-637.

5. Mylonas I, Beanlands RS. Radionuclide imaging of viable myocardium: is it underutilized? Curr Cardiovasc Imaging Rep. 2011;4:251-261.

6. Skillen A, Currie G, Wheat J. Thermal control of brown adipose tissue in ${ }^{18}$ F FDG PET. J Nucl Med Technol. 2012;40:99-103.

7. Israel O, Weiler-Sagie M, Rispler S, et al. PET/CT quantitation of the effect of patient-related factors on cardiac ${ }^{18}$ F-FDG uptake. J Nucl Med. 2007;48:234-239.

8. Fares A. Winter cardiovascular diseases phenomenon. N Am J Med Sci. 2013; 5:266-279.

9. Hopstock LA, Barnwtt AG, Bonaa KH, Mannsverk J, Njolstad I, Wilsgaard T. Seasonal variation in cardiovascular disease risk factors in a subarctic population: the Tromso Study 1979-2008. J Epidemiol Community Health. 2013;67:113-118.

10. Charach G, Shochat M, Argov O, et al. Seasonal changes in blood pressure: cardiac and cerebrovascular morbidity and mortality. World J Hypertens. 2013; $3: 1-8$.

11. Okada M, Kakehashi M. Effects of outdoor temperature on changes in physiological variables before and after lunch in healthy women. Int J Biometeorol. March 6, 2014 [E-pub ahead of print].

12. Rosenthal T. Seasonal variations in blood pressure. Am J Geriatr Cardiol. 2014;1:267-272.

13. Mias C, Coatrieux C, Denis C, et al. Cardiac fibroblasts regulate sympathetic nerve sprouting and neurocardiac synapse stability. PLOS ONE. 2013;8:e79068.

14. Vaseghi M, Lux RL, Mahajan A, Shivkumar K. Sympathetic stimulation increases dispersion of repolarization in humans with myocardial infarction. Am J Physiol Heart Circ Physiol. 2012;302:H1838-H1846.

15. Vaseghi M, Shivkumar K. The role of the autonomic nervous system in sudden cardiac death. Prog Cardiovasc Dis. 2008;50:404-419.

16. Tatsumi M, Engles JM, Ishimori T, Nicely O, Cohade C, Wahl RL. Intense ${ }^{18} \mathrm{~F}-$ FDG uptake in brown fat can be reduced pharmacologically. J Nucl Med. 2004;45:1189-1193. 